\title{
High serum carbonic anhydrase IX predicts shorter survival in head and neck cancer
}

\author{
Rosenberg $\mathrm{V}^{1}$, Pastorekova $\mathrm{S}^{2}$, Zatovicova $\mathrm{M}^{2}$, Vidlickova $\mathrm{I}^{2}$, Jelenska L ${ }^{2}$, Slezak $\mathrm{P}^{3}$ \\ Department of Radiation Oncology, Faculty Hospital with Policlinic of JA Reiman in Presov, Presov, Slovakia. \\ viktorrosenberg@gmail.com
}

\section{ABSTRACT}

OBJECTIVES: The objective of the study was to investigate prognostic and predictive value of pretreatment soluble carbonic anhydrase IX (CAIX) blood serum concentration in patients with locally advanced head and neck cancer. BACKGROUND: Increased expression of CA IX in tumor tissues has been associated with treatment resistance and worth prognosis. Soluble form of CA IX, released from tumor cells, is detectable in blood serum and could be a convenient predictive factor of treatment effectiveness that would enable treatment individualization. METHODS: The prospective study evaluated 48 patients with locally advanced squamous cell carcinomas of head and neck, treated by radiotherapy or chemo-radiotherapy. Pretreatment soluble CA IX serum concentrations were examined using enzyme-linked immunosorbent assay.

RESULTS: Soluble CA IX serum concentration failed to predict radiotherapy effectiveness in the studied patient population $(p=0.26)$. However, high CA IX serum concentrations have been associated with shorter overall survival $(p=0.035)$

CONCLUSION: High pretreatment CA IX serum concentration is a negative prognostic factor in locally advanced head and neck cancer patients (Tab. 1, Fig. 2, Ref. 23). Text in PDF www.elis.sk.

KEY WORDS: carbonic anhydrase IX, hypoxia, radiotherapy, head and neck cancer, survival.

\section{Introduction}

Despite remarkable advances in radiation oncology in recent decades, head and neck cancer still remains a serious medical and social problem. Up to $60 \%$ of cases are diagnosed in advanced stages, causing anatomical deformities and functional defects. Despite modern radiotherapy techniques, combined with concurrent chemotherapy or biological treatment, long term local control rates in advanced stages remain as low as 20 to $30 \%$ (1). Such aggressive therapy, on the other hand, is associated with substantial acute and late toxicity in virtually all of these patients which increases their morbidity and decreases their quality of life.

Tumor hypoxia represents a well-documented cause of radiotherapy resistance and treatment failure in head and neck cancer. Radiotherapy in patients with hypoxic tumors achieves inferior local control and shorter overall survival compared to well oxygenated tumors (2).

Over the years, different methods of overcoming the tumor hypoxia problem have been studied in clinical trials, including

${ }^{1}$ Department of Radiation Oncology, Faculty Hospital with Policlinic of JA Reiman in Presov, Presov, Slovakia, ${ }^{2}$ Institute of Virology, Slovak Academy of Science, Bratislava, Slovakia, and ${ }^{3}$ Education Department of Simulation and Virtual Medical Education, Faculty of Medicine, Comenius University, Bratislava, Slovakia

Address for correspondence: V. Rosenberg, MD, Department of Radiation Oncology, Faculty Hospital with Policlinic of Ján Adam Reiman in Presov, Holleho 14, SK-081 81 Presov, Slovakia.

Phone: +421.903679342 , Fax: +421.51 .7722247$ radiation with heavy particles, hyperbaric oxygen, hypoxic radiosensitizers, altered fractionation, carbogen breathing, and nicotinamide administration $(3,4)$. Most of these procedures, however, are very complex, costly and time consuming, often associated with significantly increased toxicity that disables their routine use in clinical practice. Moreover, not all patients benefit from such procedures. Hence simple and reliable method of tumor hypoxia detection and quantitative measurement is desired, that would enable patients stratification and treatment individualization.

Tumor hypoxia can be measured directly, using Eppendorf oxygen electrodes, or by administration and subsequent detection of external markers of hypoxia, such as nitroimidazoles $(5,6)$. Nonetheless, both these methods have their drawbacks and limitations. Electrode measurement is invasive and can only be performed in a limited tumor volume in anatomically accessible locations in a limited time. Administration of external markers of hypoxia also represents an invasive procedure, requiring subsequent biopsy and the acquired information is also very limited in space and time. Less invasive techniques, based on positron emission tomography or nuclear magnetic resonance imaging have significantly lower specificity and sensitivity $(7,8)$. Thus neither oxygen electrode measurement nor external hypoxia markers can be routinely applied in everyday clinical practice.

Carbonic anhydrase IX (CA IX) represents an endogenous marker of tumor hypoxia. It is detectable not only in tumor tissues but also in blood and other body fluids of cancer patients. CA IX is a zinc-containing multi-domain transmembrane metalloprotein coded by a homonymous gene. Its physiological function is 


\section{1-204}

maintaining $\mathrm{pH}$ homeostasis by catalytic reversible hydration of carbon dioxide to bicarbonate ions and protons. Under hypoxic conditions, the expression of CA IX in tumor cells rapidly increases. It represents one of the mechanisms enabling survival of tumor cells in hypoxic environment by maintaining stable intracellular $\mathrm{pH}$ and acidification of extracellular microenvironment. This process also promotes proliferation, adhesion and invasion of tumor cells $(9,10)$.

Soluble form of CA IX is a protein, released from cellular surface by proteolytic cleavage of the extracellular part of the transmembrane protein CA IX. This process is termed CA IX ectodomain shedding. Precise biological function of soluble CA IX is yet unclear but it is assumed that it might be involved in intercellular paracrine signaling. It is important that hypoxia itself does not influence the rate of CA IX ectodomain shedding, thus the amount of soluble CA IX in body fluids should reflect the amount of CA IX produced by the hypoxic cells $(11,12)$.

The amount of soluble CA IX protein in blood serum can be easily measured by enzyme-linked immunosorbent assay (ELISA). The test can be performed from a routine blood sample. Therefore soluble CA IX serum concentration, at least in theory, appears to be an ideal endogenous marker of tumor hypoxia causing radiotherapy resistance as it can be evaluated repeatedly during patient's follow-up visits and without any need of invasive procedure or special equipment.

\section{Materials and methods}

The prospective clinical study evaluated 48 patients (47 men and 1 woman), age 45-81 years, treated in the Department of Radiation Oncology at Jan Adam Reiman Faculty Hospital with Policlinic in Presov, Slovak Republic, between 2011 and 2014. All evaluated patients had histologically verified locally advanced squamous cell carcinoma of head and neck, defined as clinical stage III-IVB, according to the 7th edition of TNM Classification of Malignant Tumors (13). Patients with carcinoma of nasopharynx and carcinoma of paranasal sinuses were excluded from the study. Detailed patients characteristics are listed in Table 1.

Tab. 1. Patients characteristics.

\begin{tabular}{lccccc}
\hline Tumor location & $\begin{array}{c}\text { Oral } \\
\text { cavity }\end{array}$ & Oropharynx & $\begin{array}{c}\text { Larynx/ } \\
\text { hypopharynx }\end{array}$ & Other & Total \\
\hline Number of patients & 19 & 13 & 14 & 2 & 48 \\
\hline $\mathrm{T}_{1}{ }^{\mathrm{a}}$ & 1 & 1 & 0 & 0 & 2 \\
\hline $\mathrm{T}_{2}{ }^{\mathrm{a}}$ & 2 & 1 & 2 & 0 & 5 \\
\hline $\mathrm{T}_{3}{ }^{\mathrm{a}}$ & 6 & 6 & 9 & 0 & 21 \\
\hline $\mathrm{T}_{4}{ }^{\mathrm{a}}$ & 10 & 5 & 3 & 0 & 18 \\
\hline $\mathrm{T}_{\mathrm{x}}{ }^{\mathrm{a}}$ & 0 & 0 & 0 & 2 & 2 \\
\hline $\mathrm{N}_{0}{ }^{\mathrm{b}}$ & 2 & 2 & 3 & 0 & 7 \\
\hline $\mathrm{N}_{1}{ }^{\mathrm{b}}$ & 5 & 2 & 3 & 0 & 10 \\
\hline $\mathrm{N}_{2}{ }^{\mathrm{b}}$ & 11 & 8 & 7 & 1 & 27 \\
\hline $\mathrm{N}_{3}{ }^{\mathrm{b}}$ & 1 & 1 & 1 & 1 & 4 \\
\hline Radiotherapy alone & 3 & 3 & 0 & 1 & 7 \\
\hline Cisplatin & 5 & 4 & 11 & 1 & 21 \\
\hline Cetuximab & 11 & 6 & 3 & 0 & 20 \\
\hline
\end{tabular}

${ }^{\mathrm{a}} \mathrm{T}$ - extent of primary tumor according to TNM classification (13), ${ }^{\mathrm{b}} \mathrm{N}-$ extent of nodal involvement according to TNM classification (13)
All patients were treated with curative intent by radiotherapy as a primary treatment modality with or without concurrent chemotherapy with weekly cisplatin or biological therapy with cetuximab. The applied dose of radiotherapy was 70 Gy in 35 fractions using conventional fractionation. The decision about concurrent systemic treatment was made by the treating physician based on patient's clinical condition, regardless of patient's enrollment in study. The patients were enrolled prospectively in a consecutive order as they came to the Department of Radiation Oncology. The study was approved by the local ethics committee and all patients had signed a written informed consent form prior to their enrollment.

Blood samples were taken from all participating patients prior to the initiation of radiotherapy. $10 \mathrm{ml}$ of peripheral venous blood was driven into a standard biochemical test tube for serum examination. The blood sample was than centrifuged at 4000 revolutions per minute for 10 minutes. The obtained blood serum was frozen and temporarily stored at $-20{ }^{\circ} \mathrm{C}$. Serum concentrations of soluble CA IX were determined by sandwich ELISA, using a commercially available system from R\&D Systems, Inc., USA, catalogue number DY2188.

Treatment effectiveness was being evaluated 6 weeks after the completion of radiotherapy. Patients were clinically examined by two experts - otorhinolaryngologist and radiation oncologist. Endoscopy and radiological imaging such as ultrasonography, computerized tomography, and magnetic resonance imaging were performed according to the clinical situation. In case of uncertainty, a biopsy was performed. The median follow-up time was 30 months (range 10-48 months). Patients were divided into two categories, according to their response to radiotherapy. Complete response was defined as a complete disappearance of tumor 6 weeks after radiotherapy, with no signs of local recurrence during the subsequent follow up. Patients with tumor persistence 6 weeks after radiotherapy as well as patients with local recurrence during the follow-up, were classified as having persistent disease. In patients with persistent disease and good performance status, salvage surgery was first considered. In the inoperable cases or in case of patient's refusal of surgery, palliative chemotherapy was administered according to local standards. Patients in poor performance status were given best supportive care.

For the purpose of subgroup analysis, patients were divided into 4 subgroups according to the location of their primary tumor: Oral cavity, oropharynx, larynx/hypopharynx and other. Laryngeal and hypopharyngeal tumors were grouped into one category because in most cases both organs were affected by an advanced tumor, so it was impossible to determine where the tumor had originated from. The other subgroup included one patient with parotid gland carcinoma and one patient with malignant cervical lymphadenopathy of unknown origin.

The primary objective of the study was to determine whether pretreatment serum CA IX concentration could predict the effectiveness of radiotherapy. Secondary objective was to investigate whether pretreatment CA IX serum concentration had an impact on patient's prognosis, expressed by overall survival. We also investigated whether an association between pretreatment CA IX 
concentration and tumor location (diagnostic subgroup), tumor extent (T-category), or a degree of nodal involvement (N-category) could be found.

Data were analyzed using statistical software StatsDirect 3.0.95 and Microsoft Excel 2010. Data were normalized, using logarithmic transformation. Differences in mean CA IX concentrations between the groups were analyzed using unpaired t-test. The survival curves were generated using Kaplan-Meier analysis with the log-rank test to test for differences. Cox proportional hazard regression model was used to calculate the relative risk and hazard ratio and its $95 \%$ confidence interval in the survival analysis. All $\mathrm{p}$ values were two-sided and $\mathrm{p}<0.05$ was considered statistically significant.

\section{Results}

Among 48 evaluated patients, 16 patients (33.3\%) had complete remission of the disease, whereas 32 patients $(66.7 \%)$ had persistent disease after radiotherapy. 7 patients $(6.3 \%)$ were treated by radiotherapy only, 21 patients $(43.8 \%)$ were treated by radiotherapy with concurrent cisplatin chemotherapy, and 20 patients $(41.7 \%)$ were treated by radiotherapy with concurrent cetuximab.

Measured serum CA IX concentrations varied substantially between individual patients, ranging from 0 to $1933.44 \mathrm{pg} / \mathrm{ml}$. In the group with complete remission, the mean CA IX serum concentration was $74.07 \mathrm{pg} / \mathrm{ml}$ (range $0-393.72 \mathrm{pg} / \mathrm{ml}$ ). In the group with persistent disease, the mean serum CA IX concentration was $153.91 \mathrm{pg} / \mathrm{ml}$ (range $0-1933.4 \mathrm{pg} / \mathrm{ml}$ ). We found no statistically significant difference between the two groups $(p=0.26)$ (Fig. 1).

Median serum concentration of CA IX in the entire studied population was $28.62 \mathrm{pg} / \mathrm{ml}$. Median overall survival in the group with high CA IX concentration (above median) was 22.2 months (range 16.2-49.7 months) whereas median overall survival in the group with low CA IX concentration (below median) was 109.2 months (range 29.8-110.7 months). The difference was statistically significant $(\mathrm{p}=0.035)$, hazard ratio $2.30(1.04-5.12)$ (Fig. 2).

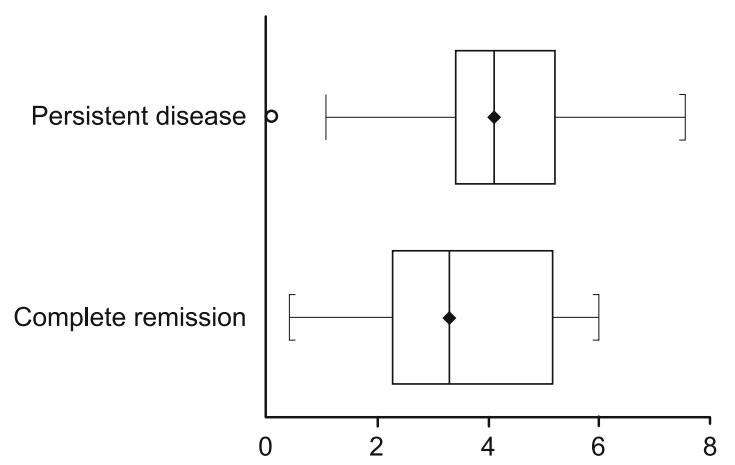

Fig. 1. Pretreatment soluble CA IX serum concentrations. Normalized data after logarithmic transformation are presented in the form of box and whiskers plot, showing pretreatment CA IX concentrations in the group with persistent disease and in the group with complete remission after radiotherapy. No significant difference between the groups was found $(p=0.26)$.

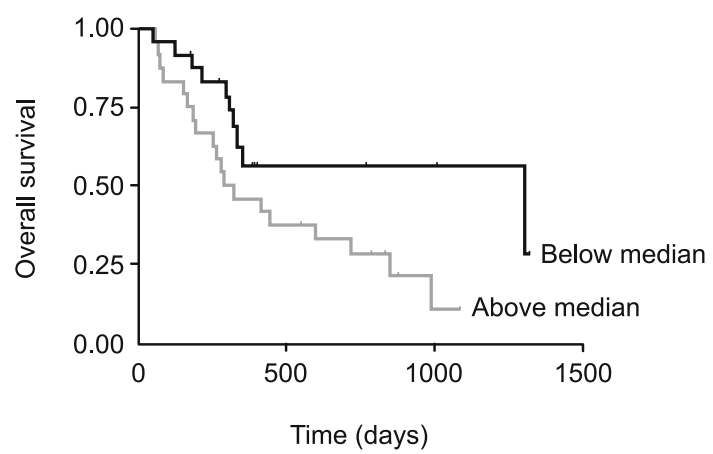

Fig. 2. Survival plot. Overall survival time of patients with low pretreatment serum CA IX concentrations (below median) was significantly longer than in patients with high CA IX concentrations (above median $)(p=0.035)$.

We also investigated whether there were differences in serum CA IX concentrations between different tumor locations, so we performed a subgroup analysis. We compared CA IX concentrations between three diagnostic subgroups (oral cavity, oropharynx, larynx/hypopharynx). We found no statistically significant difference between the subgroups $(p=0.79)$. Neither we found any association between serum CA IX concentration and T-category $(p=0.37)$ or $N$-category $(p=0.25)$ of the TNM classification. No influence of systemic therapy on the effectiveness of radiotherapy was observed in the studied patient population $(p=0.63)$.

\section{Discussion}

CA IX represents an endogenous marker of tumor hypoxia. Tumor hypoxia in head and neck cancer, measured by Eppendorf oxygen electrodes, has been associated with decreased effectiveness of radiotherapy (2). Increased expression of CA IX in many tumor types is considered to be a marker of worse prognosis. Recently published meta-analysis reported a significantly reduced overall survival and disease free survival of head and neck cancer patients with positive CA IX expression in tumor tissue samples (14). However, most of the available data comes from small retrospective studies and only few of them focused primarily on radiotherapy effectiveness.

Very little is known so far about the biological function of the soluble form of CA IX.

Increased soluble CA IX serum concentration has been associated with increased CA IX expression in tumor tissues in patients with renal cell carcinoma. It is caused by constitutional activation of the hypoxia inducible factor (HIF) pathway as a result of defective von-Hippel-Lindau (VHL) protein. In renal cell carcinoma, increased CA IX serum concentration has been associated with better prognosis and better response to treatment (15).

In other types of tumors, published clinical data investigating the prognostic value of soluble CA IX are very limited. In the surgical series, increased CA IX serum concentration before surgery has been associated with worst disease-free survival in patients 


\section{1-204}

with vulvar carcinoma (16). In patients with non-small cell lung cancer, increased CA IX plasma levels have been associated with shorter overall survival and disease specific survival, mostly in early stages (I and II) (17). On the contrary, no impact of CA IX serum concentration on the prognosis has been observed in patients with cervix (18), ovarian (19), and breast cancer (20). One study investigated plasma CA IX concentrations in patients with oral cancer. These patients exhibited significantly higher levels of soluble CA IX compared to healthy controls (21). No association between CA IX plasma levels and treatment effectiveness has been evaluated in that study. High pretreatment plasma levels of CA IX as well as other hypoxia markers were independent predictors of overall survival in non-small cell lung cancer patients treated by radiotherapy (22).

To our knowledge, this the first study investigating relation between CA IX serum concentration and survival in head and neck cancer. In our study, pretreatment serum CA IX concentration failed to predict the effectiveness of radiotherapy in locally advanced head and neck cancer patients treated by radiotherapy with curative intent. This is in agreement with our previously published data (23). These findings are in contrast with the reports evaluating the predictive role of CA IX expression in tumor tissues. However, high pretreatment CA IX serum concentration was associated with significantly shorter overall survival. No correlation between CA IX serum concentration and tumor location, tumor extent or lymph node involvement was found. CA IX serum concentration thus remained an independent prognostic factor of overall survival in the studied patient population. The clinical application of these findings could be in treatment individualization and patient selection for specific therapies such as hypoxia targeting treatments.

\section{Learning points}

High level of carbonic anhydrase IX in blood serum is associated with shorter overall survival in locally advanced head and neck cancers treated by radiotherapy.

\section{References}

1. Šlampa P, Petera J (Eds). Radiační onkologie. Praha: Galén, 2007: 457.

2. Nordsmark, Bentzen SM, Rudat V et al. Prognostic value of tumor oxygenation in 397 head and neck tumors after primary radiation therapy. An international multi-center study. Radiother Oncol 2005; 77 (1): 18-24.

3. Overgaard J. Hypoxic radiosensitization: adored and ignored. J Clin Oncol 2007; 25 (26): 4066-4074.

4. Gunderson LL, Tepper JE (Eds). Clinical radiation oncology. Philadelphia: Saunders 2012: 1638.

5. Horsman, MR. Measurement of tumor oxygenation. Int J Radiat Oncol Biol Phys 1998; 42 (4): 701-704.

6. Raleigh JA, Dewhirst MW, Thrall DE. Measuring Tumor Hypoxia. Semin Radiat Oncol 1996; 6 (1): 37-45.
7. Halmos GB, Bruine de Bruin L, Langendijk JA, van der Laan BF, Pruim J, Steenbakkers RJ. Head and neck tumor hypoxia imaging by 18F-fluoroazomycin-arabinoside (18F-FAZA)-PET: a review. Clin Nucl Med 2014; 39 (1): 44-48.

8. Bernstein JM, Homer JJ, West CM. Dynamic contrast-enhanced magnetic resonance imaging biomarkers in head and neck cancer: potential to guide treatment? A systematic review. Oral Oncol 2014; 50 (10): 963-970.

9. Pastorekova S, Parkkila S, Zavada J. Tumor-associated carbonic anhydrases and their clinical significance. Adv Clin Chem 2006; 42: 167-216.

10. Sedlakova O, Svastova E, Takacova M, Kopacek J, Pastorek J, Pastorekova S. Carbonic anhydrase IX, a hypoxia-induced catalytic component of the pH regulating machinery in tumors. Front Physiol 2014; 4: 1-10.

11. Zatovicova M, Pastorekova S. Modulation of cell surface density of carbonic anhydrase IX by shedding of the ectodomain and endocytosis. Acta Virol 2013; 57 (2): 257-264.

12. Pastorek J, Pastorekova S. Hypoxia-induced carbonic anhydrase IX as a target for cancer therapy: From biology to clinical use. Semin Cancer Biol 2015; 31: 52-64.

13. Sobin LH, Gospodarowicz MK, Wittekind C (Eds). TNM Classification of malignant tumours. Chichester: Wiley-Blackwell, 2010: 336.

14. Peridis S, Pilgrim G, Athanasopoulos I, Parpounas K. Carbonic anhydrase-9 expression in head and neck cancer: a meta-analysis. Eur Arch Otorhinolaryngol 2011; 268 (5): 661-670.

15. Zhou GX, Ireland J, Rayman P, Finke J, Zhou M. Quantification of carbonic anhydrase IX expression in serum and tissue of renal cell carcinoma patients using enzyme-linked immunosorbent assay: prognostic and diagnostic potentials. Urology 2010; 75 (2): 257-261.

16. Kock L, Mahner S, Choschzick $M$ et al. Serum carbonic anhydrase IX and its prognostic relevance in vulvar cancer. Int J Gynecol Cancer 2011; 21 (1): 141-148.

17. Ilie M, Mazure NM, Hofman V et al. High levels of carbonic anhydrase IX in tumour tissue and plasma are biomarkers of poor prognostic in patients with non-small cell lung cancer. Br J Cancer 2010; 102 (11): 1627-1635.

18. Woelber L, Kress K, Kersten JF et al. Carbonic anhydrase IX in tumor tissue and sera of patients with primary cervical cancer. BMC Cancer 2011; 11: 1-10.

19. Woelber L, Mueller V, Eulenburg $C$ et al. Serum carbonic anhydrase IX during first-line therapy of ovarian cancer. Gynecol Oncol 2010; 117 (2): 183-188.

20. Schütze D, Milde-Langosch K, Witzel I et al. Relevance of cellular and serum carbonic anhydrase IX in primary breast cancer. J Cancer Res Clin Oncol 2013; 139 (5): 747-754.

21. Yang JS, Chen MK, Yang SF et al. Increased expression of carbonic anhydrase IX in oral submucous fibrosis and oral squamous cell carcinoma. Clin Chem Lab Med. 2014; 52 (9): 1367-1377.

22. Ostheimer C, Bache M, Güttler A, Kotzsch M, Vordermark D. A pilot study on potential plasma hypoxia markers in the radiotherapy of nonsmall cell lung cancer. Osteopontin, carbonic anhydrase IX and vascular endothelial growth factor. Strahlenther Onkol 2014; 190 (3): 276-282.

23. Rosenberg V, Pastoreková S, Zat'ovičová M. Relation between carbonic anhydrase IX serum level, hypoxia and radiation resistance of head and neck cancers. Klin Onkol 2014; 27 (4): 269-275.

Received July 12, 2015, Accepted August 5, 2015. 